\title{
Amplification of weak signals and stochastic resonance via on-off intermittency with symmetry breaking
}

\author{
Changsong Zhou ${ }^{1}$ and C.-H. Lai ${ }^{1,2}$ \\ ${ }^{1}$ Department of Computational Science \\ and ${ }^{2}$ Department of Physics \\ National University of Singapore, Singapore 119260
}

\begin{abstract}
Nonlinear dynamical systems possessing reflection symmetry have an invariant subspace in the phase space. The dynamics within the invariant subspace can be random or chaotic. As a system parameter changes, the motion transverse to the invariant subspace can lose stability, leading to on-off intermittency. Under certain conditions, the bursting behavior is symmetry-breaking. We demonstrate the possibility of observing multiplicative noise(chaos)-induced amplification of weak signal and stochastic resonance via on-off intermittency with symmetry breaking in a general class of symmetrical systems. Differences of this mechanism of stochastic resonance to that in noisy bistable or threshold systems are discussed
\end{abstract}

PACS number(s): 05.40.-a, 05.45.-a 


\section{Introduction}

The phenomenon of stochastic resonance has been a subject of great interest since it was first proposed in the study of the geophysical dynamics [1]. The idea is that a signal can be amplified by the dynamics of a system in the presence of noise. The most frequently studied system is the motion of a particle in a symmetric double-well potential that is subjected to a periodic modulation and a Gaussian white noise. The amplitude of the modulation is so small that, by itself, it cannot induce any transition between the two potential wells. On the other hand, the added white noise can induce such transitions and controls the time-scale of the tunneling between the two potential wells. A resonance occurs when this time-scale matches the time period of the modulation. This phenomenon has been explored in various fields and many new applicability have been discoved, see Ref. [2] for a review of the phenomenon and further references. In general, three basic ingredients are required for a system to display stochastic resonance, including a form of threshold or energy barrier, a weak coherent input and a noise source which is inherent in the system or added to the weak input. This source of "noise" can be some form of chaotic dynamics in deterministic systems, because deterministic chaos resembles the features of noise on a coarse-grained time-scale. With these features, the system can display increased sensitivity to the weak input at an optimal noise level.

In this paper, we present a new mechanism for realizing stochastic resonance in a general class of dynamical system with reflection symmetry. Due to this symmetry, the system possess an invariant subspace in its phase space. We are interested in the case where the motion within this subspace is random or chaotic. As a system parameter changes, the stability of the subspace can be altered and the system can display unusual dynamical behaviors, among which are on-off intermittency [3, 4, 5, 6, 7, 8] and bubbling [9, 10, 11, 12]. In onoff intermittency, the invariant manifold is slightly unstable, and the system can remain close to the invariant manifold for long periods of time, interrupted only by some occasional large bursts away from the invariant manifold. In bubbling the invariant manifold is stable; however, there are unstable invariant sets embedded in the chaotic sets. As a result, small perturbations can result in large intermittent bursts from the invariant manifold. Another phenomenon that may accompany the onset of on-off intermittency is symmetry-breaking, in which the resulting bursting behavior does not possess the system symmetry, so that the system have two coexisting symmetrical attractors. However, clear symmetry breaking may not be observed in experiments because the system close to the onset point is very sensitive to external perturbations, such that any small noise in practice will induce transition of the trajectories between the two symmetrical attractors, and symmetry is restored.

In this paper, we first study how small noise affects the transition in the system. Then we investigate the response of the system to the input of a stream of very weak binary signal, periodic or aperiodic. This weak input can also induce transition of the trajectory between the two symmetrical components. In the symmetry breaking region, the transition is determined by the applied weak input, and the output can be regarded as an amplification of the weak signal. The random or chaotic motion within the invariant subspace affects the response time of the system as well as the bursting frequency. As a parameter of this motion changes, we can observe the phenomenon of stochastic resonance. Since the source of noise from the random or chaotic motion is multiplicative to the motion transverse to the invariant subspace, we call the resonant phenomenon multiplicative noise(chaos)-induced stochastic resonance. We employ various measures to characterize this phenomenon in the context of different possible applications, including residence-time distribution, bit error probability and 
amplification factor.

\section{The System}

We consider the following general class of systems:

$$
\begin{aligned}
x_{n+1} & =f\left(x_{n}\right), \\
y_{n+1} & =F\left(x_{n}, p\right) G\left(y_{n}\right),
\end{aligned}
$$

where $f(x)$ is a noise generator or a chaotic process. The function $G(y)$ possesses the symmetry $G(-y)=-G(y)$, thus the subspace $y=0$ is invariant. $F\left(x_{n}, p\right)$ is a certain coupling function which can be regarded as a multiplicative driving to the subsystem $y$, and $p$ is a tunable parameter of the driving. The stability of the invariant subspace $y=0$ is determined by the transverse Lyapunov exponent

$$
\lambda=\lim _{N \rightarrow \infty} \frac{1}{N} \sum_{n=1}^{N} \ln \left|F\left(x_{n}, p\right) G^{\prime}(0)\right|=\langle\ln |F(x, p)|\rangle,
$$

where $G^{\prime}(0)=d G(y) /\left.d y\right|_{y=0}$ is a constant. By absorbing this constant into the function $F\left(x_{n}, p\right)$, one can always set $G^{\prime}(0)=1$. Let $D_{x}=\left\langle(\ln |F(x, p)|-\lambda)^{2}\right\rangle$ denotes the variance of $\ln |F(x, p)|$. The stability of the invariant subspace is determined by the parameter $p$, and $\lambda=0$ defines the critical value $p_{c}$ which is the onset point of on-off intermittency.

\subsection{Brownian motion model for on-off intermittency and bubbling}

To understand the unusual behavior of the system close to the critical point of the stability, let us examine the linear dynamics of $y$ close to the invariant solution $y=0$, e.g. $|y| \leq \tau$, namely,

$$
y_{n+1}=F\left(x_{n}, p\right) y_{n} .
$$

A state $|y| \leq \tau$ is referred to as a laminar phase and $|y|>\tau$ a bursting phase. Here $\tau$ is a small enough value so that the linear approximation in Eq. (4) is valid. Introducing the variable $z=\ln |y|$, we get

$$
z_{n+1}=z_{n}+\lambda+\sqrt{D_{x}} \xi_{n}
$$

where $\xi_{n}=\left(\ln \left|F\left(x_{n}, p\right)\right|-\lambda\right) / \sqrt{D_{x}}$ is a random or chaotic variable with a mean 0 and a variance 1 . Now if we rescale the system to a coarse-grained time-scale by a factor of $N$ as

$$
z_{n}^{\prime}=\frac{z_{n N}}{N}, \quad \xi_{n}^{\prime}=\frac{1}{N} \sum_{i=0}^{N-1} \xi_{n N+i},(n=0,1,2, \cdots),
$$

we can see that

$$
z_{n+1}^{\prime}=z_{n}^{\prime}+\lambda+\sqrt{D_{x}} \xi_{n}^{\prime}
$$

which has the same dynamics as Eq. (5). If the random or chaotic signal $x_{n}$ has very short correlation time, $\xi_{n}^{\prime}$ will have an asymptotic Gaussian distribution for large $N$ according to the central limit theorem. In this context, the driving signal $x_{n}$ can be viewed as a kind of multiplicative noise to the motion of $y_{n}$. 
Based on the above consideration, to analyze the long time behavior, map (5) can be replaced by the corresponding stochastic differential equation

$$
\frac{d z}{d t}=\lambda+\sqrt{D_{x}} \xi
$$

where $\xi$ is a Gaussian white noise with a normal distribution $N(0,1)$. This equation describes one-dimensional Brownian motion with a constant drift $\lambda$ and a diffusion coefficient $D_{x} / 2$. When $\lambda$ is slightly positive, the motion drifts to the positive direction, leading to repulsion from the invariant subspace, but the diffusion may make the motion access deeply into the negative values of $z$, so that the system can come in and remain close to the invariant subspace for some long period of time, leading to on-off intermittency. For $\lambda$ slightly negative, the motion will eventually drift to $z \rightarrow-\infty$; however, if there is small noise added to $y_{n}$, the motion is prevented from drifting to $z \rightarrow-\infty$. The effects of the additive noise to Eq. (2) can be modeled by a reflecting boundary condition [4, 13] of the Brownian motion in Eq. (8). With this reflecting boundary, $z$ may access positive values due to the diffusion, leading to the behavior of attractor bubbling. In the presence of perturbations, on-off intermittency and bubbling are essentially the same phenomenon, and we shall refer to on-off intermittency from now on. When focusing on the laminar phases, the nonlinearity of the function $G$ is not important. It only serves to keep the state $y$ bounded.

\subsection{Symmetry breaking and conserving}

The phenomenon of symmetry-breaking, however, is associated with the nonlinearity of the function $G$. In Fig. 1, we show two possible situations of the system behavior. Let $Y_{1}>0$ and $Y_{2}>0$ the values of $y$ at which $G\left(Y_{1}\right)=\max [|G(y)|]$ and $G\left(Y_{2}\right)=0$. Plot (a) depicts the situation where $y_{\max }=\max [|F(x, p)|] G\left(Y_{1}\right)<Y_{2}$, so that a trajectory starting with $0<y_{0}<Y_{2}\left(-Y_{2}<y_{0}<0\right)$ will remain in the positive (negative) part forever in the noisefree case, and the system is symmetry-breaking since the trajectory does not possess the reflection symmetry of the function $G$. While in plot (b), $y_{\max }>Y_{2}$, and the system has only one asymptotic attractor possessing the reflection symmetry, thus is symmetry-conserving. A transition from one symmetrical component to the other happens whenever $\left|y_{n}\right|>Y_{2}$. The point where $y_{\max }=Y_{2}$ is a symmetry increasing bifurcation point [14].

To study the property of the symmetrical dynamical system subject to small noise or coherent signal, we employ a function $G$ in which $Y_{2}$ is adjustable independently. Since many properties observed in the following are quite common in this class of systems, we choose in this paper a piecewise linear function for simplicity of analysis.

$$
G(y)= \begin{cases}\frac{1}{c}(-1-c-y), & y<-1 \\ y, & |y| \leq 1 \\ \frac{1}{c}(1+c-y), & y>1\end{cases}
$$

Also since the properties of on-off intermittency are universal for many different driving signals $F\left(x_{n}, p\right)$, we simply let $F\left(x_{n}, p\right)=p x_{n}$, and use uniform random numbers $x_{n} \in(0,1)$ in simulations. With this implementation of the system, one has $\lambda=\ln p-1, D_{x}=1$, $y_{\max }=p$ and $Y_{2}=1+c$ so that the symmetry increasing bifurcation occurs at $c_{s}=p-1$. The onset point of on-off intermittency is $p_{c}=e=2.71828 \ldots$

In the following several simulations, we take $p=2.8$ above the critical point if not explicitly pointed out. Fig. 2 shows typical behavior of the system with symmetry breaking $(c=2)$ and symmetry conserving $(c=1)$. 


\subsection{Sensitivity to small perturbations}

Before we demonstrate the sensitivity of the system to weak coherent signal and the phenomenon of stochastic resonance, we show how the system behaves in the presence of additive small noise. Small noise sets a reflecting boundary to the Brownian motion and can change the property of the laminar phases considerably [4, [13]. Noise has another effect on the system behavior: it can induce in the trajectory transitions between the two symmetrical components of the system, and the symmetry breaking will not manifest. As an example, Fig. 3 shows system behavior corresponding to Fig. 2, but with an additive small noise

$$
y_{n+1}=p x_{n} G\left(y_{n}\right)+e_{n} .
$$

The standard deviation of the Gaussian white noise $e_{n}$ is $\delta=10^{-5}$. In the presence of even very small noise, symmetry is restored for the originally symmetry-breaking system. Since in practice noise is inevitable, clear symmetry breaking as in Fig. 2(a) cannot be observed in real experiments.

The bursting patterns in Fig. 3(a) and (b) are qualitatively different. In Fig. 3(a), transition between $y>0$ and $y<0$ occurs only when $y$ come to the noise level, while in Fig. 3 (b) transition can occur both in laminar phases and bursting phases.

\section{Amplification of weak signal and stochastic resonance}

In most previous studies on stochastic resonance, the noise induces hopping between the states separated by a barrier or a threshold with an average waiting time $\left\langle T_{r}\right\rangle$. When $\left\langle T_{r}\right\rangle$ is comparable with half the period of the applied weak signal, this noise-induced hopping becomes statistically synchronized with the weak signal, and stochastic resonance ensues. In our system, the mechanism is different. We do not need additive noise to generate transitions. A weak signal by itself can generate such transitions.

Let us first consider the response of the system to a weak periodic binary signal:

$$
\begin{aligned}
y_{n+1} & =p x_{n} G\left(y_{n}\right)+s_{n}, \\
s_{n+2 T} & =s_{n}=\left\{\begin{aligned}
A, & 0<n \leq T, \\
-A, & T<n \leq 2 T .
\end{aligned}\right.
\end{aligned}
$$

In the example in Fig. 4, we can see astonishing difference of the system response to a weak periodic signal with amplitude $A=10^{-5}$ and bit duration $T=1000$. It is clear that for the symmetry-breaking case, the transition is now almost totally governed by the switching of the periodic binary signal. A weak sinusoidal input will produce similar output. While for the symmetry-conserving system, there are many additional transitions induced by bursting phases in the duration of a signal bit, and the output do not have the clear periodicity of the signal.

To characterize the difference in the bursting pattern, we calculate the distribution of the residence time. The residence time $T_{r}$ is defined as follows: starting with a moment $n_{0}$ when the system produces for the first time a large bursting state, say $y_{n_{0}}<-y_{t h}, n_{1}$ is the subsequent time when the system first produce a bursting state $y_{n_{1}}>y_{t h}$ and $n_{2}$ is the time when $y_{n_{2}}<-y_{t h}$ again, and so on; the quantity $T_{r}(i)=n_{i}-n_{i-1}$ represents the residence time the system stays in one of the symmetrical component between two subsequent 
transition events. $y_{t h}$ is a value of the order of $y_{\max }$. In all our simulations in this paper, we set $y_{t h}=1.0$.

Fig. (5) shows the residence time distribution $P\left(T_{r}\right)$ of the bursting behavior in Fig. (3). For the symmetry-breaking system, $T_{r}$ distributes around the bit duration $T$ of the input signal. While for the symmetry conserving system, the distribution is almost the same as that of the input of noise (not shown).

We should point out that for symmetry-breaking system, a single peak of $P\left(T_{r}\right)$ around $T$ occurs only for $T$ large enough. For $T$ smaller than a certain value, the distribution $P\left(T_{r}\right)$ begins to show other peaks centered at odd multiples of the bit duration $T$, i.e. at $(2 k-1) T(k=1,2 \ldots)$, and the smaller the $T$, the greater the number of peaks. The peak height decreases exponentially with $k$. This property is quite common in stochastic resonance systems [2]. Fig. 6 shows the results of $P\left(T_{r}\right)$ for $T=1000$ and $T=200$. For $T=1000$, a very small peak at $k=2$ begins to show up, while for $T=200$, seven peaks are clearly discernible. This behavior is associated with the relaxation time $T_{0}$ of the system, which is the time for the system to relax to the statistically stationary state after the signal switches from one value to the other. When $T_{0}>T$, the transition of the system is not able to keep up with the switching of the signal. The appearance of the peaks can be simply explained: the system may perform a transition after the signal switches from a bit to the other, thus $T$ is a preferred residence time. If the system fails to perform a transition before the signal switches back, it has to wait for a full period before it can produce a transition, and the second peak is therefore located at $3 T$, and so on.

The behavior of the system with a weak signal is determined by the competition between the strength of the constant drift $\lambda$ and the diffusion $D_{x} / 2$. If $p$ is far away below the critical point, the system will quickly relax to a signal-induced metastability state, and can rarely produce large bursting states, and the bursting pattern may not possess the periodicity of the weak signal. With $p$ approaching $p_{c}$, the system can produce large bursting states more frequently, and the periodicity of the signal can be manifested. When $p$ is far away above the critical point, the system can seldom have access to the level of weak input $|y| \sim A$, and the switching of the signal can no longer determine the transition of $y$; the bursting pattern loses the periodicity of the signal again. An optimal response will be obtained around the critical point where the drift is dominated by the diffusion, and the system can have access to the level of the weak input so that the transition is sensitive to the switching of the weak signal, and at the same time can produce large bursting states quite quickly.

In order to quantify the the response of the system as a function of $p$, we introduce the area under the peak centered at the bit duration $T$ in the residence time distribution as a measure of the strength of the periodicity:

$$
S=\sum_{T-T / 4}^{T+T / 4} P\left(T_{r}\right) .
$$

$S$ as a function of $p$ is shown in Fig. 7. It increases with $p$, reaches a maximum and decreases again, displaying the typical feature of stochastic resonance. This feature is quite robust to noise additive to the weak periodic signal, as seen in Fig. 7 where $S$ as a function of $p$ is also shown for different level of additive noise. When $p$ increases, the system may enter into the symmetry-conserving region, and the transition during the bursting phases will degrade the periodicity of the output. Now if $c$ has such a value that the system enters into the symmetry-conserving region before reaching the resonant point, the maximal value of $S$ will 
be slightly after the symmetry increasing point $p_{s}=1+c$, because the transition induced by the bursting phases is rare just beyond this point, and it become more significant for $p$ going deeper into the symmetry converging region. An example of this case is shown in Fig. 7 for $c=1.5$, where the maximal $S$ is found around $p=2.55$. This optimal response is not a resonant behavior in the sense discussed above. However, in a wider sense, it can also be regarded as a resonant phenomenon because it results from the competition between the transition induced by the weak signal and that induced by the bursting phases.

In the above, we characterize the stochastic resonance by the residence time distribution for a periodic binary signal. One should have noticed that the periodicity of the signal is not important for observing the resonance phenomenon. However, if we consider aperiodic signals, for example a random series of binary bits, we should employ some other quantities to quantify the phenomenon.

First, let us consider a possible application of the system in the detection of a weak signal with bit duration $T$. The detection is performed as follows: we look at the large bursting state $|y|>y_{t h}$; if the sum of these states in a bit duration is positive (negative), then this bit is detected as $+1(-1)$; if this number is zero, we cannot make a decision. With this scheme, one may detect a very weak signal with a low-resolution detector. We calculate the probability of bit error $P_{e}$ as a function of $p$. The result is shown in Fig. 8. A random stream of signal with $10^{6}$ bits is used in simulation. When $p$ is quite below the critical point, the system produces large bursts quite sporadically, and only a very small portion of the bits is detected. The bit error probability is close to 1.0. On the opposite hand, the system produces large bursting states quickly, but is not sensitive to the signal, and $P_{e}$ tends to 0.5. An optimal detection with smallest bit error probability is obtained around the critical point. We also calculate $P_{e}$ in the presence of additive noise. Again we see that the system is very robust to additive noise. In this sense, the system may find application in the detection of a weak signal buried in relatively high level of noise.

Next let us consider the application of the system as an amplifier of the weak signal. A natural measure of the output is the ensemble average $\left\langle y_{n}\right\rangle$. An amplification factor $I$ can be defined as

$$
I^{2}=\lim _{N \rightarrow \infty} \frac{1}{N} \sum_{n=1}^{N} \frac{\left\langle y_{n}\right\rangle^{2}}{A^{2}} .
$$

For a periodic signal,

$$
I^{2}=\frac{1}{T} \sum_{n=1}^{T} \frac{\left\langle y_{n}\right\rangle^{2}}{A^{2}} .
$$

For this system, we can give an analytical estimation of the amplification factor under the adiabatic condition $T \gg T_{0}$. To perform this analysis, let us recall the Brownian motion model in Eq. (8). The probability distribution of the variable $z$ satisfies the Fokker-Planck equation

$$
\frac{\partial W}{\partial t}=-\lambda \frac{\partial W}{\partial z}+\frac{D_{x}}{2} \frac{\partial^{2} W}{\partial z^{2}} .
$$

The small signal and confinement of the nonlinearity can be modeled by two reflecting boundaries of the Brownian motion. If $T \gg T_{0}$, the probability distribution of $z$ will establish a stationary state during a bit duration, namely, $W(z)=C \exp (\alpha z)$, where $\alpha=2 \lambda / D_{x}$. In the original variable $y$, it becomes $W(y)=C|y|^{\alpha-1}$. If the system is symmetry breaking, after reaching the stationary state, $y$ is always positive (negative) for signal bit $+1(-1)$. With 
the normalization condition

$$
\int_{A}^{y_{e f f}} C y^{\alpha-1} d y=1,
$$

where $y_{\text {eff }}$ is a parameter used to represent the reflecting boundary due to the nonlinearity of the system, we can estimate the amplitude of the ensemble average $\left\langle y_{n}\right\rangle$ of the system with a weak input $($ bit +1$)$ as

$$
\langle y\rangle \approx \frac{\int_{A}^{y_{e f f}} y W(y) d y}{\int_{A}^{y_{e f f}} W(y) d y}=\frac{\alpha}{1+\alpha} \frac{y_{e f f} \beta-A}{\beta-1},
$$

where $\beta=\left(y_{\text {eff }} / A\right)^{\alpha}$. From Eq. (18), $\langle y\rangle$ is a monotonic increasing function of $p$ and the stochastic resonance cannot be observed should the adiabatic condition hold true for any value of $p$.

Close to the critical point $p_{c},|\alpha| \ll 1$, the diffusion is dominant and the system can produce large bursting states quickly. As an estimation, we can just take $y_{\text {eff }}=y_{\max }$. If $|\alpha| \ln \left(y_{\max } / A\right) \ll 1$, one has $\beta \approx 1+\alpha \ln \left(y_{\max } / A\right)$. For weak input $A \ll y_{\max }$, we arrive at

$$
\langle y\rangle \approx \frac{y_{\max }}{\ln \left(y_{\max } / A\right)},
$$

which decreases to zero with the decrease of the signal amplitude $A$ only logarithmically. The amplification factor in this case is just

$$
I=\frac{\langle y\rangle}{A}=\frac{y_{\max }}{A \ln \left(y_{\max } / A\right)},
$$

which shows that the system is very sensitive to weak signal close to the critical point. This feature of sensitivity is quite different from that of the sensitivity near the onset of a perioddoubling bifurcation in many dynamical systems [15]. There the system is only sensitive to perturbations near half the fundamental frequency of the system for bifurcation parameter very close to the onset point.

This model analysis is demonstrated by simulation. Fig. 9 shows $I$ as a function of $m$ for small signal amplitude $A=10^{-m}$. The parameter $p=2.72$ in this simulation is very close to the critical point and $T \gg T_{0}$ is satisfied. It is seen that the analytical estimation of Eq. (20) with $y_{\max }=p$ fits the simulation result very well for $A$ covering several orders.

If the adiabatic condition is not satisfied, the system may not establish a stationary state during a bit duration, and the transient behavior during the relaxation process becomes significant. The time-dependent probability distribution is not easy to obtain, and we rely on simulations to estimate $I$. Although the measure $I$ is applicable to aperiodic signals, we employ periodic signal in our simulation, because it can reduce considerably the computation effort.

Now let us investigate how $I$ changes with $p$. As stated in the above, if $p$ is far away below the critical point, the constant drift $\lambda=\ln p-\ln p_{c}<0$ makes the system stay most of time close to the low boundary $|y| \sim A$. The amplitude of the ensemble average is quite small. It increases quickly when $p$ approaches the critical point where the diffusion become dominant. However, $T_{0}$ is also increasing, and $\left\langle y_{n}\right\rangle$ may not reach the maximal value in Eq. (18) when the system is not quick enough to follow the modulation of the signal, and $I$ may begin to decrease. There will be an optimal amplification of the signal. Since the relaxation time is longer for smaller $A$, the stochastic resonance peak shifts to smaller value of $p$ for 
smaller $A$. The amplification will be degraded further if the system enters into the symmetry conserving region.

Typical behavior of the system with respect to the parameter $p$ is illustrated in Fig. 10 . In this simulation, we take $c=2.2$ so that the system will move into the symmetry-conserving region at $p_{s}=1+c=3.2$ which is high above the critical point. Fig. 10(a) shows time series of $\left\langle y_{n}\right\rangle$ for several typical values of $p$. The dependence of $I$ on the parameter $p$ is shown in Fig. 10(b) for different values of $c$ and signal amplitude $A$. Complying with the above analysis, $I$ increases with $p$ untill $T_{0}$ becomes long enough or untill the system moves into the symmetry-conserving region. We will observe the optimal system response to the small signal in the region $p<p_{s}$ if $T_{0}$ becomes significantly longer than $T$ before the system enters into the symmetry-conserving region, as for the case $A=10^{-7}$ in Fig. 10 (b) where the maximal $I$ is found around $p=2.95$ before $p_{s}=3.2$. The maximum will be slightly after the point $p_{s}$ if the system enters into the symmetry-conserving region before reaching the resonant point, as for the case of $A=10^{-5}$ and $c=1.8$ in Fig. 10(b).

Again we examined the robustness of the property of stochastic resonance to additive noise. In Fig. 11, I as a function of $p$ for different level of additive noise is shown. Amplification of the small signal is obtained even if the noise level is much higher than the signal amplitude.

Since amplification of weak signal and stochastic resonance in the system can be understood by the Brownian motion model derived from the linear dynamics close to the invariant subspace, this phenomenon is universal in a general class of symmetrical systems with random or chaotic motion within the subspace.

\section{Discussion}

We demonstrate a new mechanism of stochastic resonance in a general class of symmetrical dynamical systems with on-off intermittency and symmetry breaking. The system has an invariant subspace whose stability is determined by the random or chaotic motion within the subspace. Close to the critical point of the stability, the system is very sensitive to small perturbations, since the state $y$ has a power-law distribution in a wide interval $10^{-m}<|y|<$ $y_{\text {eff }}$. The behavior of the system response to a weak binary signal can be understood by the competition between the drift and the diffusion in the Brownian motion model derived from the linear dynamics closed to the invariant subspace. When the diffusion becomes dominant for the parameter $p$ close to the critical point of stability, the system attains the ability of amplification of a very weak signal, not via additive noise, but via multiplicative noise. When the parameter $p$ determining the stability varies across the critical point from below to above, the sensitivity to the weak signal increases and then decreases after reaching a maximum, displaying the phenomenon of stochastic resonance. The resonance occurs when the system can have access to the level of the weak signal to become sensitive to the switching of the signal, and produce large output quickly at the same time.

There are several differences of this mechanism of stochastic resonance to that in a noisy bistable or threshold system.

(1) The source of noise is not additive but multiplicative, which is inherent from the random or chaotic motion within the invariant subspace.

(2) In a noisy bistable or threshold system, the weak signal by itself cannot induce hopping over the the energy barrier or the threshold. The additive noise induces such tunneling 
process which introduces a noise-controlled time-scale into the system. When this timescale matches that of the weak signal, the statistical synchronization of the tunneling to the signal leads to resonant response to the signal in the system. In the present mechanism of stochastic resonance, the multiplicative noise does not induce transition between the two symmetrical component, and there does not exist a noise-induced time scale of the transition. The transition is induced by the weak signal itself in the symmetry breaking case. A change of the level of the multiplicative noise changes the response time of the system. The resonance occurs not because this response time matches the period of the signal, but because the system responds quickly enough to follow the switching of the signal and produces large output quickly after a switching. The resonant phenomenon is not restricted to periodic signals. We have employed different quantities rather than the conventional signal-to-noise ratio suitable for a sinusoidal input to quantify the phenomenon.

(3) In our systems with symmetry breaking, although there exist two distinguished symmetrical parts in the system, there is not any clear form of threshold or barrier in the system.

The phenomenon demonstrated in this paper is universal in a class of systems. Many systems, for example symmetrically coupled identical chaotic systems [12, 16, 17, 18] or interacting stranger attractors [19], can be reduced to a form similar to the systems discussed in this paper, and we may expect to observe similar phenomenon around the critical point of synchronization. However, in such systems, a change of the parameter of the coupling strength may change the chaotic dynamics within the synchronization manifold, and this can bring about additional complication into the system response to a weak signal. This will be a topic for future study.

The behavior of on-off intermittency or bubbling may be harmful in some applications, such as secure communication using synchronization of chaos [20], because in practice highquality synchronization can be destroyed by large intermittent bursts from the synchronization manifold due to unavoidable perturbations [9, 10, 11, 12]. We demonstrate that such behavior, however, can be employed to amplify extremely weak signal, and the amplification is shown to be very robust to additive noise. This may lead to useful applications of the behavior which is quite universal in many systems.

\section{Acknowledgements:}

This work was supported in part by research grant No. RP960689 at the National University of Singapore. Zhou is supported by NSTB. 


\section{References}

[1] R. Benzi, A. Sutera, and A. Vulpiani, J. Phys. A 14, L453 (1981).

[2] L. Gammaitoni, P. Hänggi, P. Jung, and F. Marchesoni, Rev. Mod. Phys. 70, 223 (1998).

[3] N. Platt, E. A. Spiegel, and C. Tresser, Phys. Lett. 70, 279 (1993).

[4] N. Platt, S. M. Hammel, and J. F. Heagy, Phys. Rev. Lett. 72, 3498 (1994).

[5] J. F. Heagy, N. Platt, and S. M. Hammel, Phys. Rev. E 49, 1140 (1994).

[6] Y. H. Yu, K. Kwak, and T. K. Lim, Phys. Lett. A 198, 34 (1995).

[7] A. Cenys, A. Namajunas, A. Tamasevicius, and T. Schneider, Phys. Lett. A 213, 259 (1996).

[8] H. L. Yang and E. J. Ding, Phys. Rev. E 54, 1361 (1996).

[9] P. Ashwin, J. Buescu, and I. Stewart, Phys. Lett. A 193, 126 (1994).

[10] J. F. Heagy, T. L. Carroll, and L. M. Pecora, Phys. Rev. E 52, R1253 (1995).

[11] D. J. Gauthier and J. C. Bienfang, Phys. Rev. Lett. 77, 1751 (1996).

[12] S. C. Venkataramani, B. R. Hunt, E. Ott, D. J. Gauthier, and J. C. Bienfang, Phys. Rev. Lett. 77, 5361(1996).

[13] A. Cenys, and H. Lustfeld, J. Phys. A 29, 11 (1996).

[14] Ying-Cheng Lai, Phys. Rev. E 53, R4267, (1996).

[15] K. Wiesenfed and B. McNamara, Phys. Rev. Lett. 55, 13 (1985).

[16] T. Yamada and H. Fujisaka, Prog. Theor. Phys. 70, 1240 (1983).

[17] L. M. Pecora and T. L. Carroll, Phys. Rev. Lett. 64, 821 (1990).

[18] K. Josic, Phys. Rev. Lett. 80, 3053 (1998).

[19] A. S. Pikovsky, Z. Phys. B 55, 149 (1984).

[20] K. M. Cuomo and A. V. Oppenheim, Phys. Rev. Lett. 71, 65 (1993). 


\section{Figure Captions}

Fig. 1. Illustration of two cases in the system: (a) $y_{\max }<Y_{2}$, symmetry breaking and (b) $y_{\max }<Y_{2}$, symmetry conserving.

Fig. 2. Typical time series of on-off intermittency. (a) $c=2$, symmetry breaking and (b) $c=1$, symmetry conserving.

Fig. 3. Typical time series of on-off intermittency in the presence of small noise with $\delta=10^{-5}$. (a) $c=2$, symmetry breaking and (b) $c=1$, symmetry conserving.

Fig. 4. Typical time series of on-off intermittency in the presence of weak periodic signal. with amplitude $A=10^{-5}$ and bit duration $T=1000$. (a) $c=2$, symmetry breaking and (b) $c=1$, symmetry conserving.

Fig. 5 Residence time distribution $P\left(T_{r}\right)$ in the presence of weak periodic signal with $A=10^{-5}$ and $T=1000$. Plot 1: $c=2$, symmetry breaking; plot 2: $c=1$, symmetry conserving.

Fig. 6. Residence time distribution $P\left(T_{r}\right)$ in the presence of weak periodic signal with $A=10^{-5}$ but different bit duration $T$.

Fig. 7. The strength $S$ of the first peak as a function of $p$ for different level of additive noise. The input signal is $A=10^{-7}, T=1000$.

Fig. 8. Bit error probability $P_{e}$ as a function of $p$. The parameters are $c=3.0, A=$ $10^{-7}, T=1000$.

Fig. 9. Amplification of the weak signal with $A=10^{-m}$ under the adiabatic condition $T \gg T_{0}$. The amplification factor (dots) is obtained by the ensemble average estimated with $10^{4}$ samples of the time series of the system at $p=2.72$. The solid line is the analytical result of Eq. (20) with $y_{\max }=p$.

Fig. 10. Amplification property as a function of the parameter $p$. (a) Time series of the ensemble average $\left\langle y_{n}\right\rangle$ for several typical values of $p$. (1) $p=2.4$, (2) $p=3.1$, and (3) $p=3.4$. Other parameters are $A=10^{-5}, T=1000$ and $c=2.2$. (b) $I$ as a function of $p$ for different values of $A$ and $c . T=1000$.

Fig. 11. Robustness of the stochastic resonance to additive noise. $A=10^{-7}, T=1000$. 


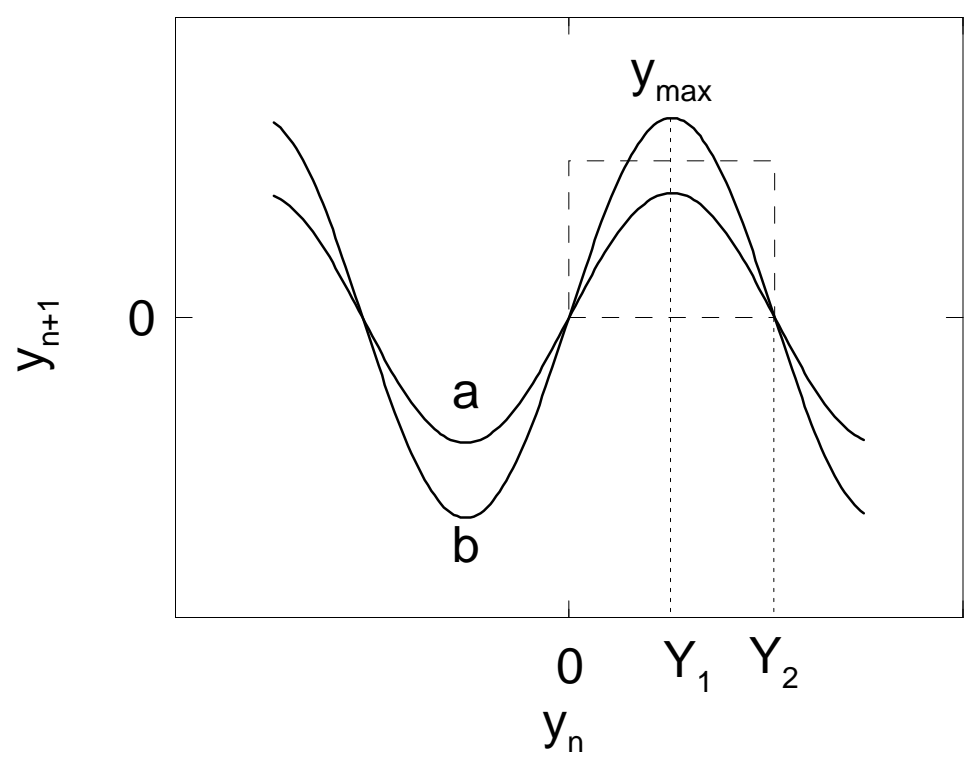

Fig. 1 


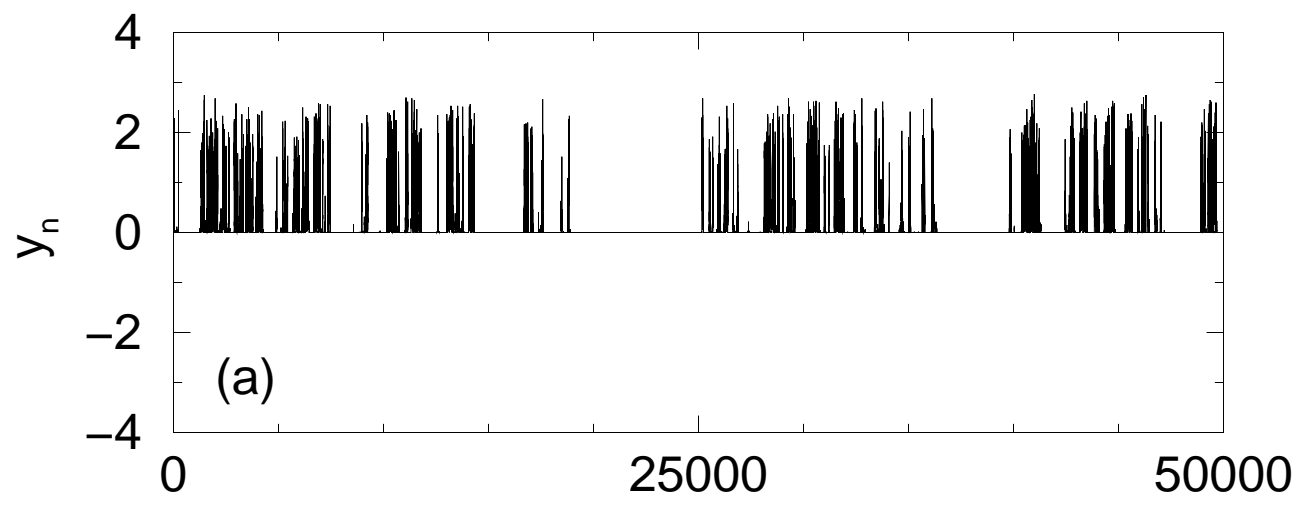

Fig. 2

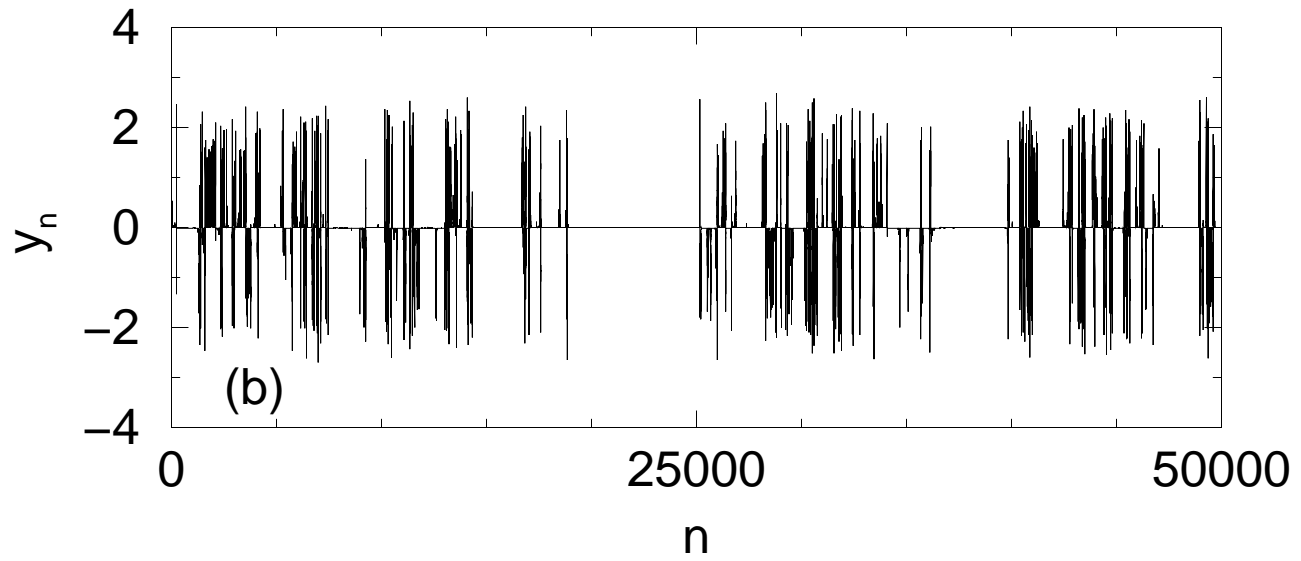




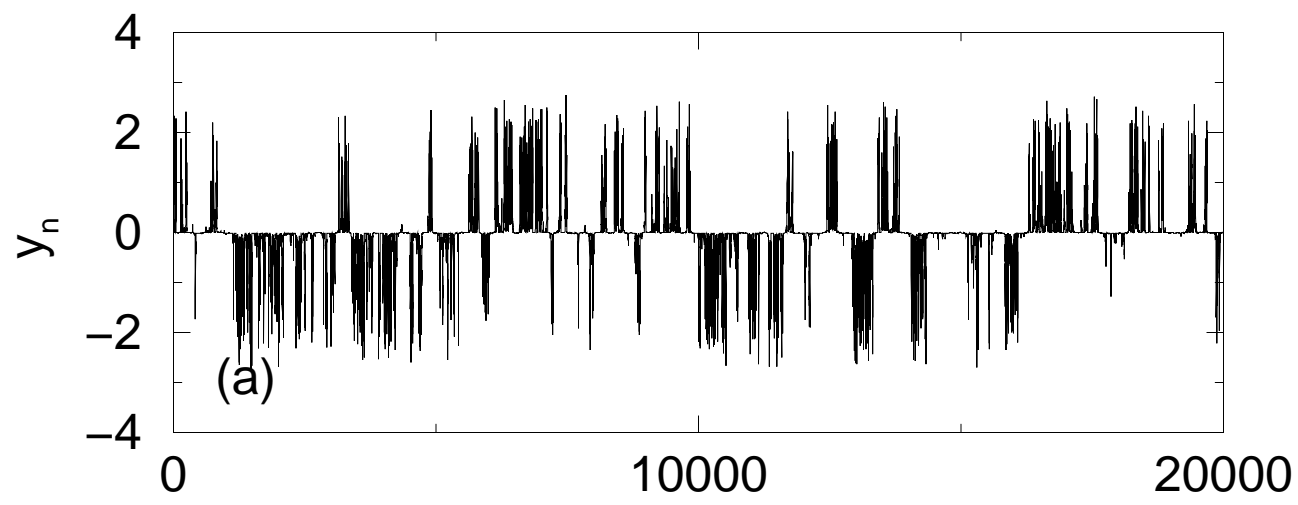

Fig. 3

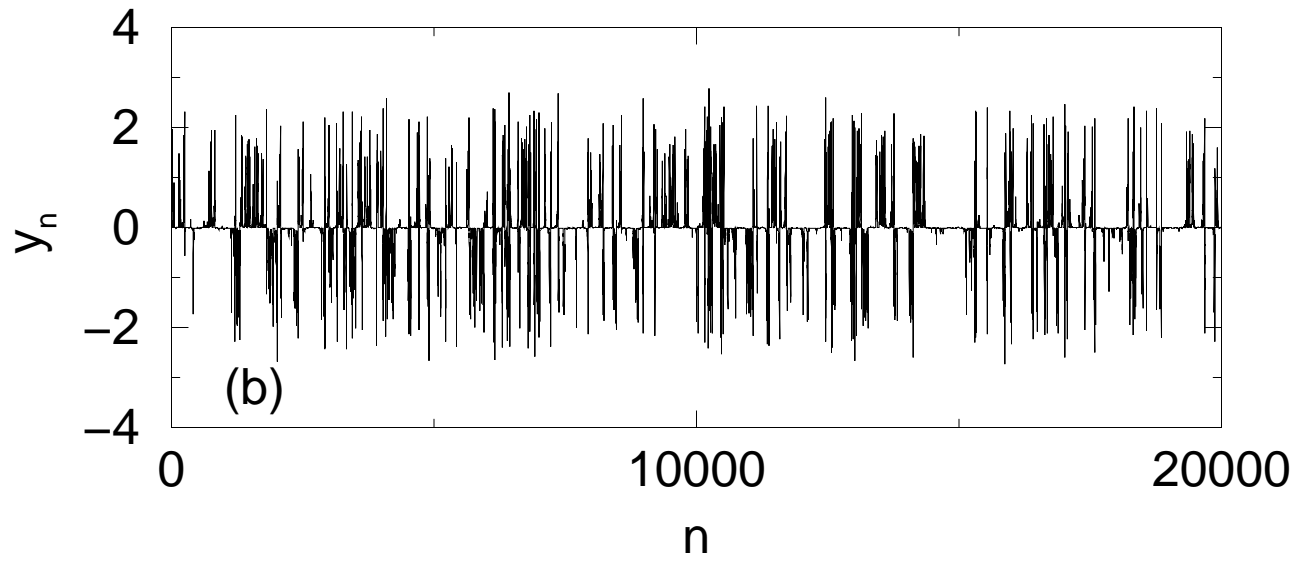




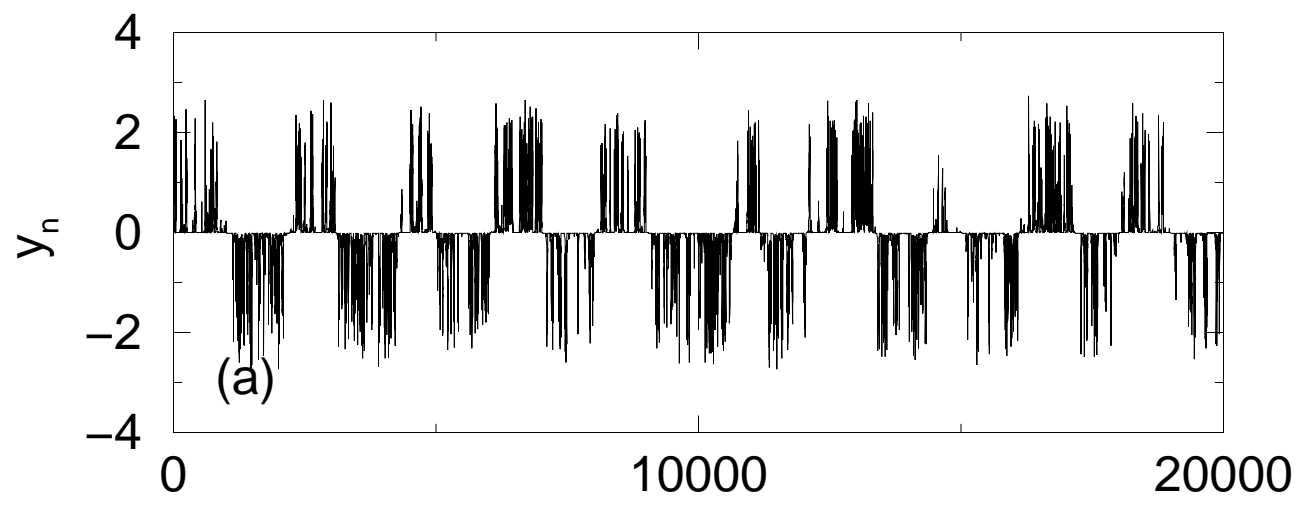

Fig. 4

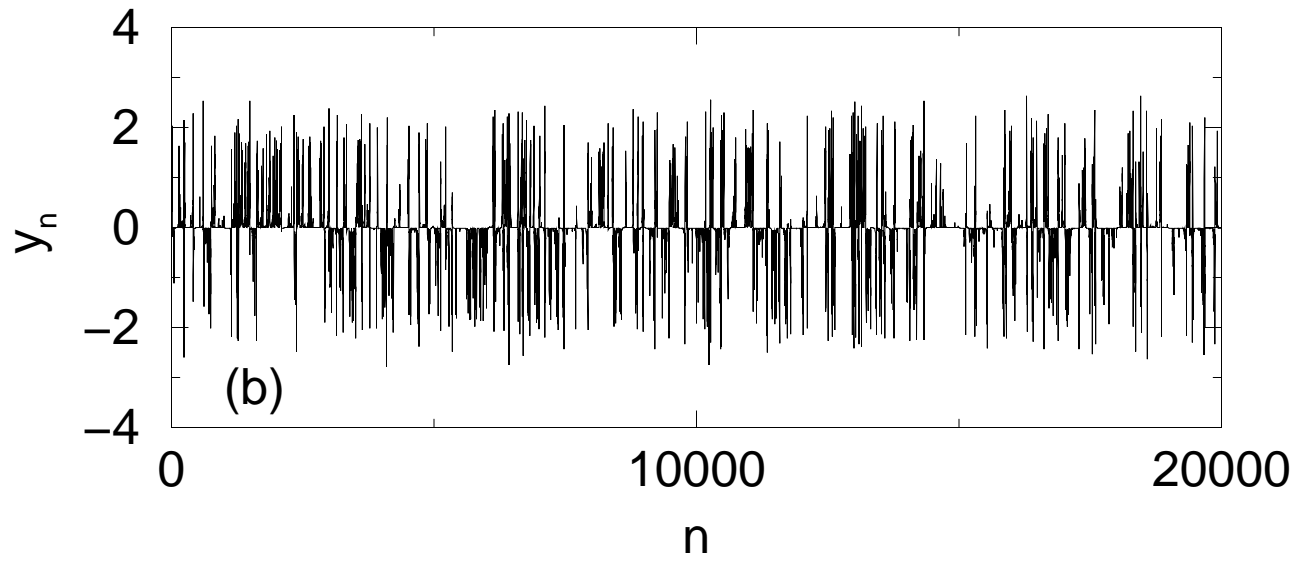




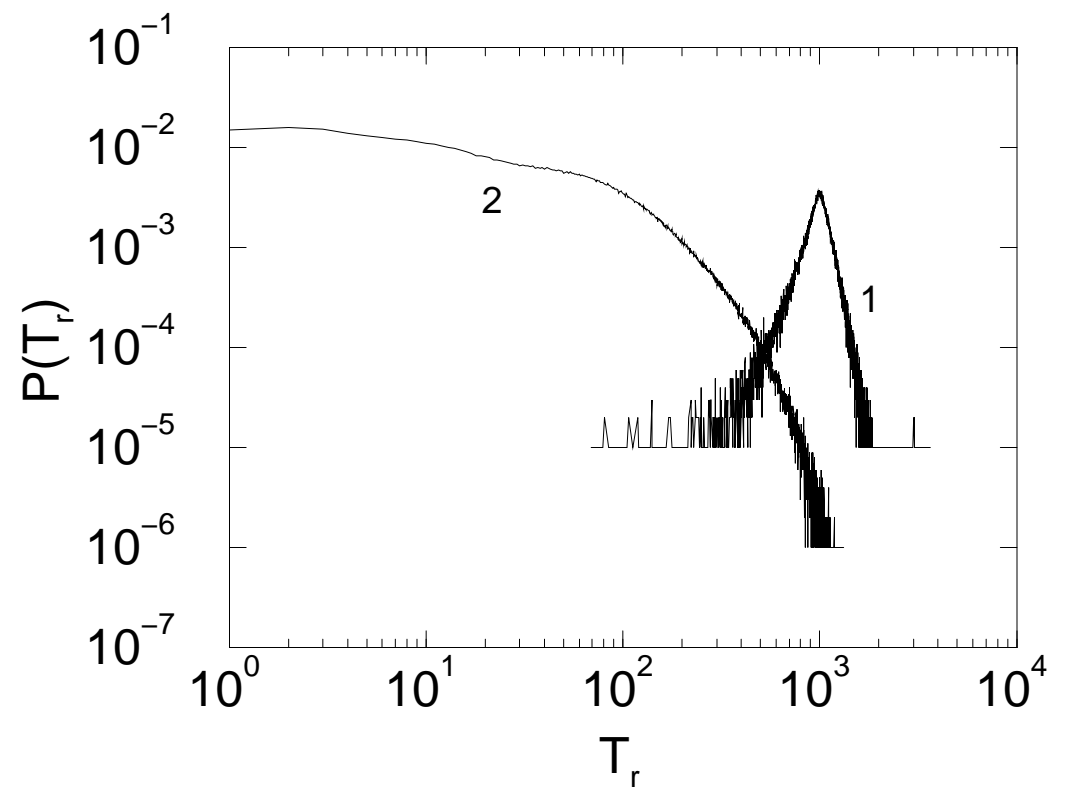

Fig. 5 

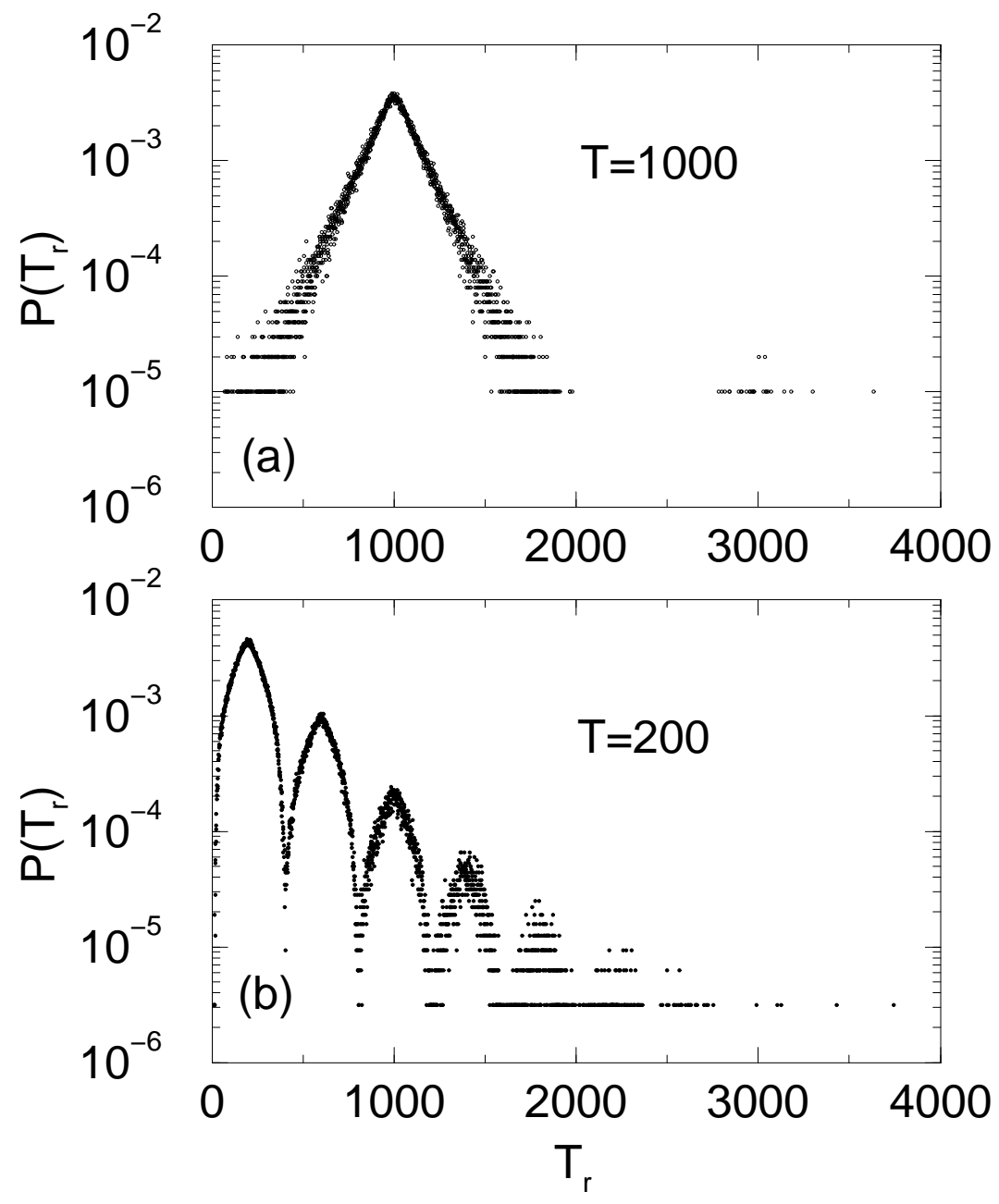

Fig. 6 


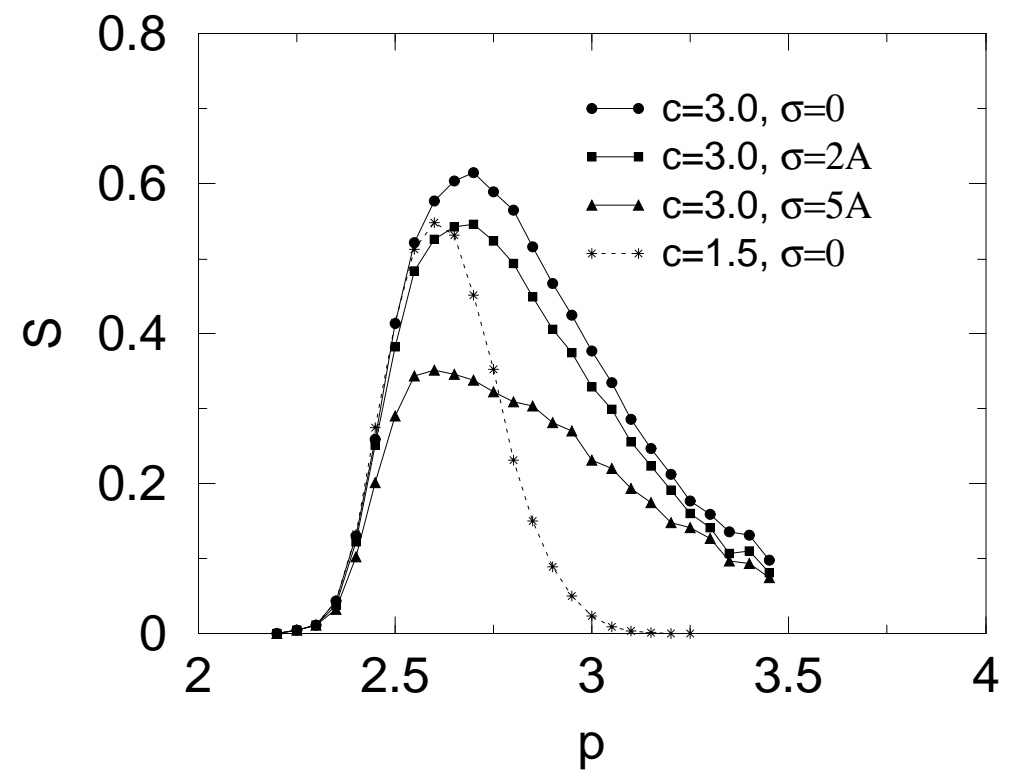

Fig. 7 


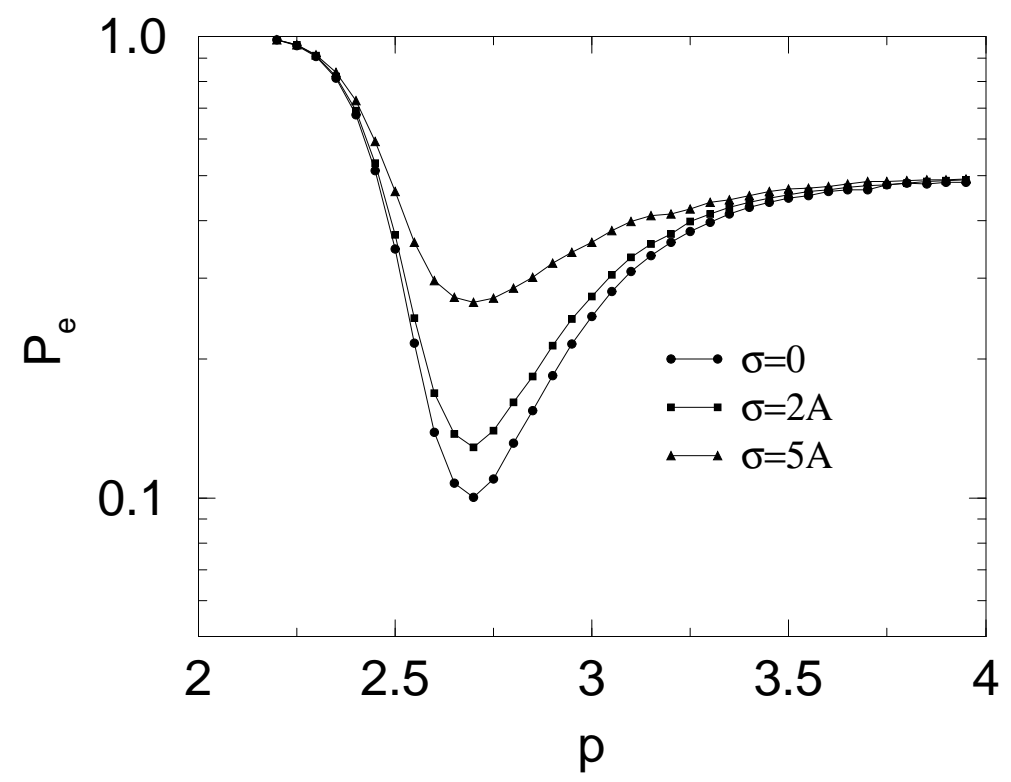

Fig. 8 


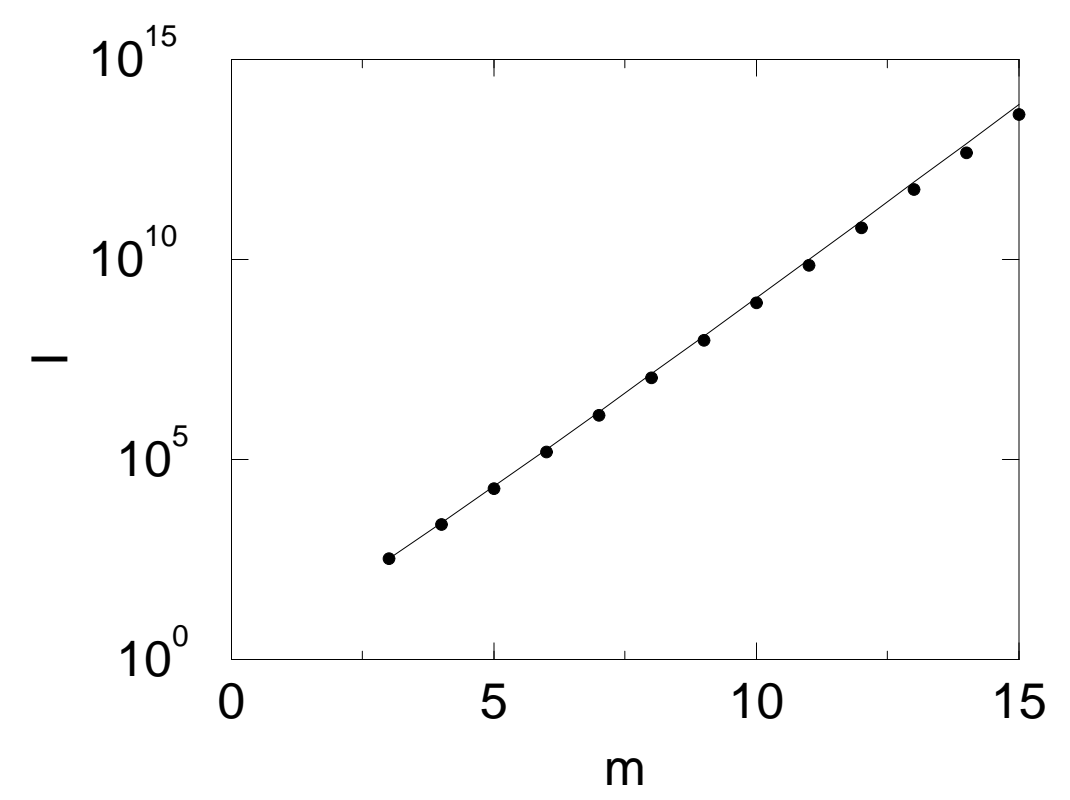

Fig. 9 
Fig. 10
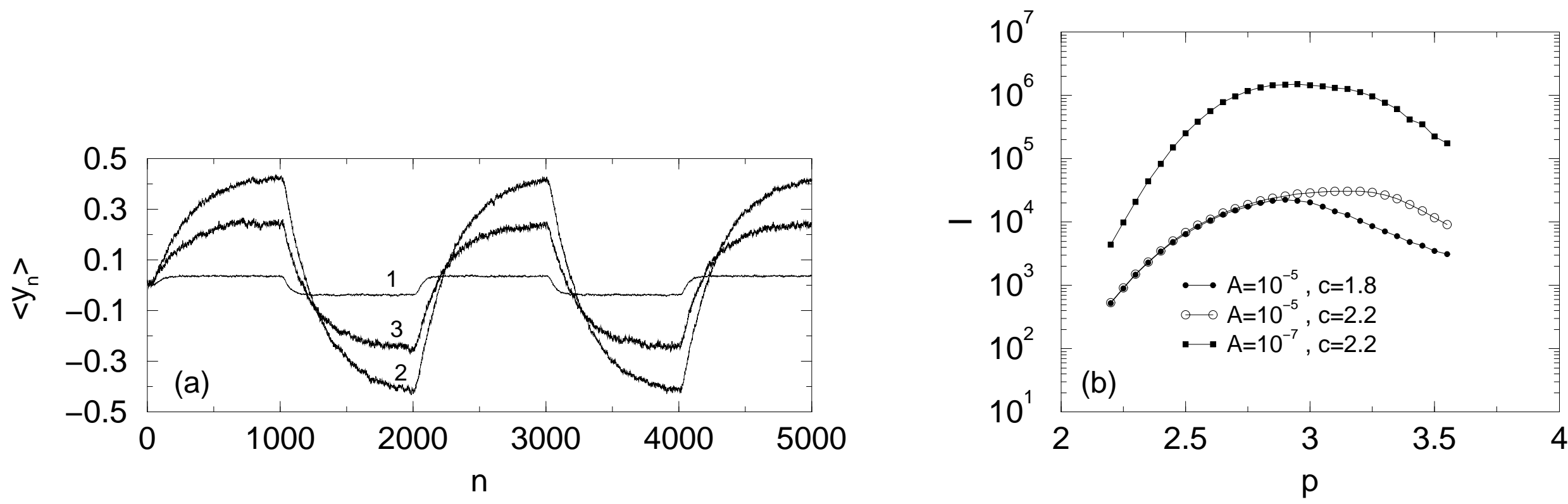


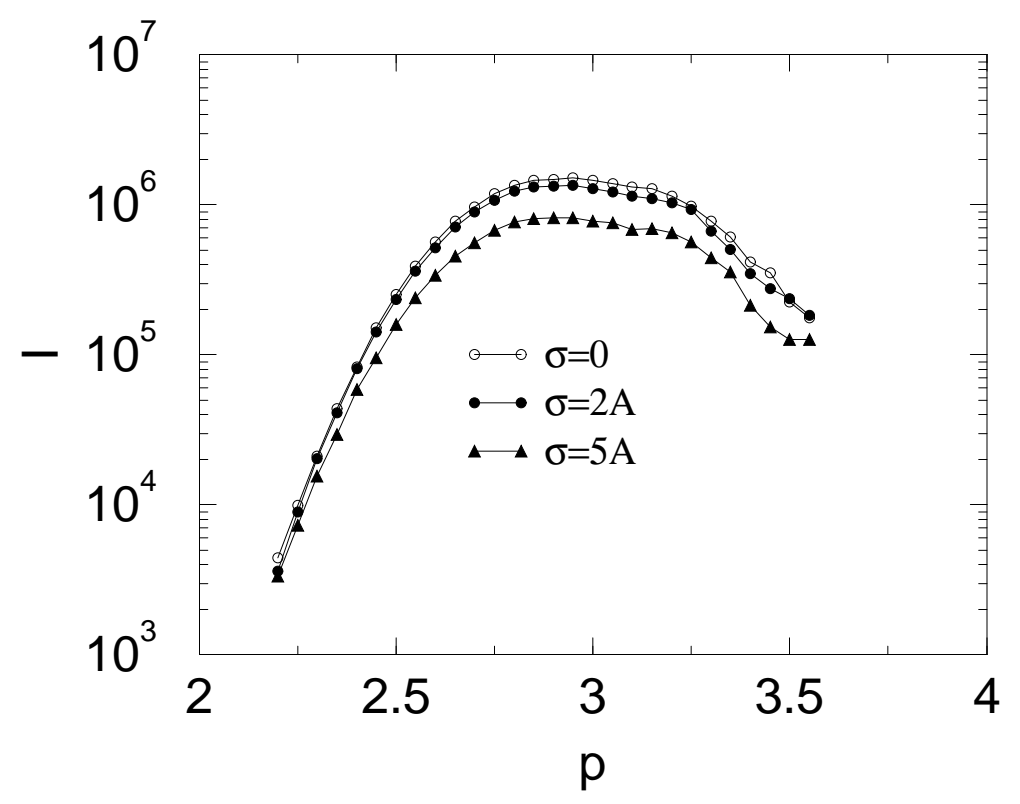

Fig. 11 